\title{
Virus-Like Particles Harboring CCL19, IL-2 and HPV16 E7 Elicit Protective T Cell Responses in HLA-A2 Transgenic Mice
}

\author{
Victoria Juarez ${ }^{1, \#}$, H. Amalia Pasolli ${ }^{2}$, Andrea Hellwig ${ }^{3}$ Natalio Garbi ${ }^{4}$ and Angel Cid Arregui ${ }^{*, 5}$ \\ ${ }^{I}$ European Molecular Biology Laboratory, Meyerhofstrasse 1, D-69117 Heidelberg, Germany \\ ${ }^{2}$ Laboratory of Mammalian Cell Biology and Development, The Rockefeller University, New York, USA \\ ${ }^{3}$ Department of Neurobiology, Interdisciplinary Center for Neurosciences (IZN), University of Heidelberg, Im \\ Neuenheimer Feld 364, D-69120 Heidelberg, Germany \\ ${ }^{4}$ Department of Molecular Immunology, Institutes of Molecular Medicine and Experimental Immunology (IMMEI), \\ University of Bonn, Sigmund Freud Str. 25, D-53105 Bonn, Germany \\ ${ }^{5}$ Translational Immunology, German Cancer Research Center (DKFZ), Im Neuenheimer Feld 460, D-69120 Heidelberg, \\ Germany
}

\begin{abstract}
Infection by high-risk genotypes of human papillomaviruses (HR-HPVs) is the cause of cancer of the uterine cervix. Although prophylactic vaccines directed against the two most prevalent HR-HPV types (HPV16 and 18) have been commercialized recently, there is a need for effective therapeutic vaccines against HR-HPVs. We have tested in mice a chimeric protein composed of the hepatitis B small surface antigen $(\mathrm{HBsAg}(\mathrm{S}))$ flanked at its $\mathrm{N}$-terminus by chemokine CC ligand 19/macrophage inflammatory protein-3 $\beta$ (CCL19/MIP-3 $\beta$ ), and at the C-terminus by interleukin 2 (IL-2) and an artificial HPV16 E7 polytope. This protein is assembled into nanoparticles and both CCL19 and IL-2 conserve their functionality. HLA-A2 (AAD) transgenic mice immunized with a plasmid encoding this protein mounted specific $\mathrm{T}$ cell responses against E7 without the need of an adjuvant. Furthermore, vaccination prevented the development of tumors after implantation of the E6/E7-expressing TC-1/A2 tumor cell line. Our results suggest that vaccines based on $\mathrm{HBs} \mathrm{Ag}(\mathrm{S})$ nanoparticles carrying short E7 epitopes and immune-stimulatory domains might be of therapeutic value in the treatment of patients suffering from cervical pre-cancer or cancer lesions caused by HR-HPVs.
\end{abstract}

Keywords: HPV, cervical cancer, HBsAg, synthetic genes, therapeutic vaccine, vaccine, VLP.

\section{INTRODUCTION}

Infection of the uterine cervix with a subset of HPV types, the so-called high-risk types (HR-HPVs), is the main cause of cervical intraepithelial neoplasia (CIN), which evolves through a series of stages (cervical intraepithelial neoplasia, CIN I-III) to in situ and invasive cancers. There is also growing evidence showing that HR-HPVs are associated with a subset of head and neck cancers. Recently, prophylactic vaccines against the two most prevalent HR-HPVs (HPV16 and 18) have been developed [1]. These vaccines are made up of the major HPV capsid protein (L1) and prevent infection by these virus types, and also might cross-protect against other virus types. However, they cannot be used to treat established HPV infections, because persistently infected cells do not express the HPV capsid proteins [2]. Nevertheless, they continuously express the E6 and E7 transforming proteins encoded by HR-HPVs, which are required for the maintenance of $\mathrm{CIN}$ lesions and the progression to cancer $[3,4]$.

*Address correspondence to this author at the Translational Immunology, German Cancer Research Center DKFZ, Im Neuenheimer Feld 460, D-69120 Heidelberg, Germany; Tel: +49 6221 567109;

Fax: +496221 564773; E-mail: a.cid@dkfz.de

"Present Address: AsteriaPharma, Mannheimer Str. 37, D-68723 Oftersheim, Germany
A number of diverse strategies for the development of therapeutic vaccines that might be beneficial in the treatment of CIN and cancer caused by HPVs have been tested in preclinical and clinical studies [5-7]. The particulate nature of virus-like particles makes them suitable vehicles as vaccines. The small envelop protein of the hepatitis B virus (HBV), known as hepatitis B surface antigen-small $(\mathrm{HBsAg}(\mathrm{S})$ is an integral membrane protein with capacity to self assemble into empty particles (virus-like particles, VLPs) without participation of other viral proteins. HBsAg(S) VLPs have been used as carrier of viral envelop and capsid epitopes, antigens of the malaria parasite as well as tumor antigens. The ability of $\mathrm{HBsAg}(\mathrm{S})$ VLPs to induce $\mathrm{T}$ cell responses may be associated to the fact that they are readily endocytosed [8]. We have previously constructed and tested a recombinant adenovirus vector encoding a chimeric protein comprising the HPV16 E7 ${ }^{35}$ deletion mutant covalently linked to the $\mathrm{C}$ terminus of $\operatorname{HBSAg}(\mathrm{S})$, which generated E7-specific cytotoxic $\mathrm{T}$ lymphocyte (CTL) responses in C57BL/6 mice [9]. In this study, we hypothesized that $\operatorname{HBsAg}(\mathrm{S})$ VLPs carrying immunestimulatory domains would have enhanced anti-tumor effects. Therefore, as a proof-of-concept, we extended our immunization studies by using a complex chimeric VLPs composed of HBsAg-S carrying the CCL19/MIP-3 $\beta$ 
chemokine, IL-2 and antigenic sequences derived from the HPV16 E7 protein. We tested the immunogenicity of this fusion protein in HLA-A2 transgenic mice, since they are thought to have CTL repertoires close to those of the human. This fusion protein elicited E7-specific $\mathrm{T}$ cell responses in immunized HLA-A2 (AAD) transgenic mice. Further, immunized mice were protected from tumor growth after implantation of a HPV16 E6/E7-expressing tumor cell line.

\section{MATERIALS AND METHODS}

\section{Cells}

TC-1 tumor cells [10] were obtained from the American Type Culture Collection CRL-2785). These cells derived from a C57BL/6 mouse and were immortalized with the amphotropic retrovirus vector LXSN16E6E7 and subsequently transformed with the pVEJB plasmid expressing the activated human c-Ha-ras oncogene. TC-1/A2 cells kindly provided by $\mathrm{Dr} \mathrm{TC} \mathrm{Wu}$ (The Johns Hopkins University, Baltimore, USA), derived from TC-1 cells after infection with retrovirus produced in Phoenix cells transfected with plasmid pMSCV-HLA-A2 (AAD) [11]. Both cell lines were cultured in RPMI 1640 supplemented with $1 \mathrm{mM}$ sodium pyruvate, $100 \mathrm{IU}$ penicillin, $100 \mu \mathrm{g} / \mathrm{ml}$ streptomycin and $10 \%$ fetal bovine serum. CTLL-2 is a cytotoxic $\mathrm{T}$ cell line derived from $\mathrm{C} 57 \mathrm{BL} / 6$ inbred mice (ATCC number TBI-214) [12]. Human embryonic kidney (HEK 293T) cells growing on 12-well plates were transfected using Lipofectamine ${ }^{\circledR}$ (Invitrogen) with $1 \mu \mathrm{g}$ of the indicated plasmid/well and incubated at $37^{\circ} \mathrm{C} / 5 \% \quad \mathrm{CO}_{2}$ for 48 hours before they were processed for Western blot.

\section{Mice}

Transgenic AAD mice (HLA-A*0201/D ${ }^{\mathrm{d}}$ ) [13] were purchased from The Jackson Laboratories and were maintained in individually ventilated racks under specific pathogen-free conditions. All experimental procedures were performed in accordance with approved protocols and regulations by the Animal Protection Commission of the state of Baden-Württemberg.

\section{Immunization and Tumor Challenge Protocols}

For the immunizations we used endotoxin-free plasmid DNA (see below) prepared with the EndoFree Plasmid Maxi kit of Qiagen. The plasmids were administered to groups of 5 female mice 6-12 weeks of age via intramuscular (M. tibialis) in four doses (100 $\mu \mathrm{g}$ plasmid/dose) at intervals of 2 weeks. To monitor whether DNA immunization conferred protective immunity against E7, tumor challenge experiments were performed one week after the last immunization by injecting the mice subcutaneously (s.c.) in the inner side of the left leg with $5 \times 10^{4}$ TC-1/A2 viable cells in phosphate-buffered saline (PBS). The onset and progression of tumor growth was monitored twice a week.

\section{Plasmids and DNA Constructs}

Plasmid pcDNA3 (Invitrogen) was used to express the CHI-E7p and H-E7p genes in transient transfections of cells in culture and for the immunization of mice. Plasmid pPICZ(A) was used for expression of these genes in the yeast Pichia pastoris. Synthetic, codon-optimized sequences encoding HBsAg(S), human IL-2, CCL19/ MIP-3 $\beta$ and the HPV16 E7p polytope (encompassing E7 amino acids 11-20 and 82-90) were obtained using long oligonucleotides that were linked together and amplified by PCR as described elsewhere [14].

\section{Peptides}

The following HPV16 E7 peptides were used: E7(11-20) (YMLDLQPETT) and E7(82-90) (LLMGTLGIV), both corresponding to previously described HLA-A*0201restricted epitopes [15].

\section{Expression and Purification of Recombinant CHI-E7p Protein}

The CHI-E7 and H-E7p proteins, carrying a carboxylterminal 6x His tag, were expressed in P. pastoris using the pPICZ(A) expression plasmid, which contains the methanolinducible AOX1 promoter (EasySelect ${ }^{\circledR}$ Pichia Expression kit, Invitrogen) using the X-33 strain of $P$. pastoris. Selection of recombinant clones was made on YPD plates containing $100 \mu \mathrm{g} / \mathrm{ml}$ Zeocin $^{\mathrm{TM}}$. His-tagged proteins were purified by ion metal affinity chromatography on nickelNTA-agarose columns (Novagen) following instructions of the manufacturer. The fractions containing the recombinant proteins were desalted and concentrated using Amicon filters (Millipore). The protein concentration in the purified fractions was determined using the BCA assay (Pierce).

\section{Western Blotting}

HEK 293 cells growing on 6 well plates were transfected using Lipofectamine (Invitrogen) with $2 \mu \mathrm{g}$ of plasmid and incubated for 48 hours. The cells were then washed twice with PBS, and lysed in SDS Laemmli sample buffer containing $1 \mathrm{mM}$ DTT. The proteins were separated on 4$12 \%$ gradient polyacrylamide gels (NuPAGE, Invitrogen) polyacrylamide gels by SDS-PAGE, blotted onto PVDF membranes, blocked with 5\% dry milk in PBS containing $0.1 \%$ Tween 20 and incubated at room temperature either with anti-6xHis (Novagen), anti-CCL19 (Pharmingen), antiHBsAg or anti-IL2 antibodies followed by peroxidaseconjugated anti-mouse or anti-rabbit antibodies as required. Antibody binding was visualized with enhanced chemiluminiscence reagent (Renaissance $\AA$, NEN-Perkin Elmer).

\section{Electron Microscopy}

The ultrastructural analysis of CHI-E7p VLPs of purified fractions from yeast lysates was carried out by negative staining. In brief, $10 \mu \mathrm{l}$ drops of a purified fraction were placed on Formvar coated grids and incubated for 1 minute. After blotting off excess of sample, grids were stained with $1 \%$ uranyl acetate in water and subsequently air-dried. Samples were examined with a Tecnai G2-13 transmission electron microscope (FEI) and photographed with a digital camera (model XR60; Advanced Microscopy Techniques, Corp.).

\section{Chemoattraction Assay}

Dendritic cells (DCs) were expanded from bone marrow progenitor cells of HLA-A2 transgenic mice as described elsewhere [16] with modifications. In brief, the femoral and tibial bones of a single mouse were dissected and placed in PBS. The bone marrow cells were flushed with PBS into a centrifuge tube using a $1 \mathrm{ml}$ syringe with a $27 \mathrm{G}$ needle. After 
centrifugation at $400 \mathrm{xg}$ for $5 \mathrm{~min}$ and standard erythrocyte depletion, the cells were cultured in a $10 \mathrm{~cm}$ dish with RPMI1640 medium supplemented with $10 \%$ fetal bovine serum, $10 \mathrm{ng} / \mathrm{ml}$ granulocyte-macrophage colony-stimulating factor (GM-CSF) and $1 \mathrm{ng} / \mathrm{ml} \mathrm{IL-4} \mathrm{(Promokine).} \mathrm{The} \mathrm{yield}$ was about 4-8 $810^{6}$ cells per dish. The medium was changed on day 3 and on day 7, cells were treated overnight with TNF- $\alpha$. DCs were then isolated using CD11c microbeads (Miltenyi Biotech) and subsequently stained at a concentration of $10^{6}$ cells $/ \mathrm{ml}$ with $0.6 \mu \mathrm{M}$ carboxyfluorescein diacetate succinimidyl ester (CFSE). Chemotaxis in response to the fusion protein was determined by measurement of fluorescence intensity of the cells migrating through a polycarbonate filter $(5 \mu \mathrm{m}$ pore size $)$ in a 24 -transwell plate (Costar). DCs $\left(10^{5}\right.$ cells) were added to the upper chamber in $100 \mu \mathrm{l}$ and the protein was added to the lower chamber in $600 \mu 1$ medium X-Vivo (Lonza). After 2 hours, fluorescence intensity was measured in the lower chamber using a fluorescence plate reader (Victor ${ }^{\circledR}$, Perkin Elmer). Background fluorescence was measured in wells with no protein or irrelevant protein added to the lower chamber and the mean values subtracted from those obtained with the CHI-E7p protein.

\section{Cell Proliferation Assays}

CTLL-2 cells express constitutively IL-2 receptors and are dependent upon IL-2 for growth. The cells were seeded in 96-well plates at a concentration of $5 \times 10^{3}$ cells/well in $100 \mu \mathrm{l}$ medium containing the indicated amounts of either IL-2 (final concentration of 0.01 to $10 \mathrm{ng} / \mathrm{ml}$ ) or CHI-E7p protein. The cells were incubated for 48 hours at $37^{\circ} \mathrm{C}$ and $5 \% \mathrm{CO}_{2}$. Then, the Cell Proliferation Reagent WST-1 (Roche) was added (10 $\mu \mathrm{l} /$ well) and the cells were incubated for additional 2 hours. After that time the absorbance was measured against a background control using a Victor ${ }^{\circledR}$ microplate reader (Perkin Elmer). Measurement of formazan product was set at $450 \mathrm{~nm}$ and the reference wavelength was $650 \mathrm{~nm}$.

\section{Intracellular Cytokine Staining and In Vivo CTL Assay}

E7-specific cytotoxic activity was detected in vivo after immunizing HLA-A2 (AAD) transgenic mice four times at 2-week intervals as described [9]. Splenocytes were isolated from naïve mice and from immunized mice 7 days after the last immunization, and were restimulated in vitro by incubating the cells overnight with $1 \mu \mathrm{g} / \mathrm{ml}$ of HPV16 E7 peptide, either E7(11-20) or E7(82-90), or without peptide (control). Cytokine release was blocked by adding GolgiPlug (BD, Pharmingen) 6 hours before harvesting the cells for cytokine staining. Then, CD8 and intracellular interferongamma (IFN- $\gamma$ ) were stained and measured as described previously [9].

For the in vivo CTL assay, splenocytes isolated from donor naïve mice were pulsed either with peptide $\mathrm{E} 7^{11-20}$, $E 7^{82-90}$ or no peptide and subsequently labeled with $3 \mu \mathrm{M}$ $\left(\mathrm{CFSE}^{\text {high }}\right), \quad 0.08 \mu \mathrm{M} \quad\left(\mathrm{CFSE}^{\mathrm{low}}\right)$ and $0.5 \mu \mathrm{M} \quad\left(\mathrm{CFSE}^{\text {int }}\right)$, respectively. Equal numbers of the three labeled cell suspensions were mixed and injected intravenously in immunized mice $\left(12 \times 10^{6}\right.$ cells/mouse $)$ six days after the last immunization. The mice were sacrificed 16 hours after injection of labeled cells and splenocytes isolated and analyzed by flow cytometry. Specific lysis was calculated as described [9]. The levels of specific lysis were calculated with the formula: \% specific lysis $=[100-($ ratio primed $\mathrm{x}$ $100) /\left(\right.$ ratio unprimed)], where ratio (\% CFSE $^{\text {low }}$ or $\left.\mathrm{CFSE}^{\text {high }}\right) /\left(\% \mathrm{CFSE}^{\text {int }}\right)$.

\section{RESULTS}

\section{Design and Construction of the CCL19/HBsAg(S)/IL- 2/E7p (CHI-E7p) Synthetic Gene}

We have shown previously the superior immunogenicity of an $\mathrm{HBsAg}(\mathrm{S})-\mathrm{E} 7$ (H-E7) vaccine compared to the E7 protein alone. In order to enhance the immunogenicity of the HBsAg(S) VLPs we sought to design a new vaccine based on VLPs made of $\mathrm{HBsAg}(\mathrm{S})$ harboring two immunestimulatory domains, at its $\mathrm{N}$ - and $\mathrm{C}$-terminus. To this end, we chose to link the CC chemokine ligand 19 (CCL19) and interleukin-2 (IL-2) to the $\mathrm{N}$ - and C-terminal ends of $\operatorname{HBsAg}(\mathrm{S})$, respectively. The function of $\mathrm{T}$ lymphocytes is regulated by DCs, which act as antigen presenting cells, and depends on encounters of naïve T cells and DCs. Chemokine ligands and receptors are thought to regulate migration of DCs to draining lymph nodes $[17,18]$. CCR7 is expressed in mature DCs [19] and, upon binding to its ligand CCL19, induces their migration to lymph nodes. Proinflammatory cytokines, such as interleukin-2 (IL-2) stimulate INF- $\gamma$ secretion and proliferation of $\mathrm{T}$ cells and NK cells and potentiate their cytotoxic function. IL-2 has been approved for the treatment of patients with metastatic renal-cell carcinoma and malignant melanoma. The antitumor effect of IL-2 is likely due to its ability to expand lymphocyte populations and increase their effector functions [20].

Codon-optimized, synthetic DNA sequences encoding the human CCL 19 chemokine and IL-2 were linked to the 5'- and the 3'-ends of the $\mathrm{HBsAg}(\mathrm{S})$ gene, respectively. In addition, a sequence encoding a polytope (E7p) derived from HPV16 E7 (including two E7 epitopes comprising amino acids 11-20 and 82-90) was inserted downstream the IL-2 coding sequence. The structure of the resulting protein encoded by such chimeric gene, named CHI-E7p, and the predicted VLPs derived from it are depicted in Fig. (1). In addition, as a control, we constructed another fusion gene consisting of $\mathrm{HBs} \mathrm{Ag}(\mathrm{S})$ linked to the same E7 polytope $(\mathrm{H}-$ E7p) at its C-terminus. Both constructs carried a C-terminal 6x His Tag.

To confirm that these chimeric genes encoded the expected fusion proteins we carried out transient transfections of HEK293 cells with pcDNA3 plasmids encoding either the CHI-E7p or the H-E7p fusion proteins. Cell lysates were electrophoresed, blotted and the proteins detected with anti-6xHis antibodies 48 hours after transfection. As shown in Fig. (2a) the CHI-E7p protein was detected as a specific band of $\sim 55 \mathrm{kDa}$. A band of the same size was detected using anti-CCL19, anti-HBsAg or anti-IL2 antibodies (data not shown). The H-E7p protein appeared as a $\sim 28 \mathrm{kDa}$ band.

We have shown previously that $\mathrm{HBsAg}(\mathrm{S})$ proteins linked C-terminally to the HPV16 E7 protein, either

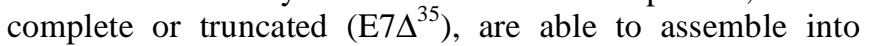
VLPs [9]. Therefore, we asked whether the CHI-E7 fusion protein also forms VLPs. Recombinant CHI-E7p protein carrying a $6 \mathrm{x}$ His Tag at its C-terminus was expressed in the 


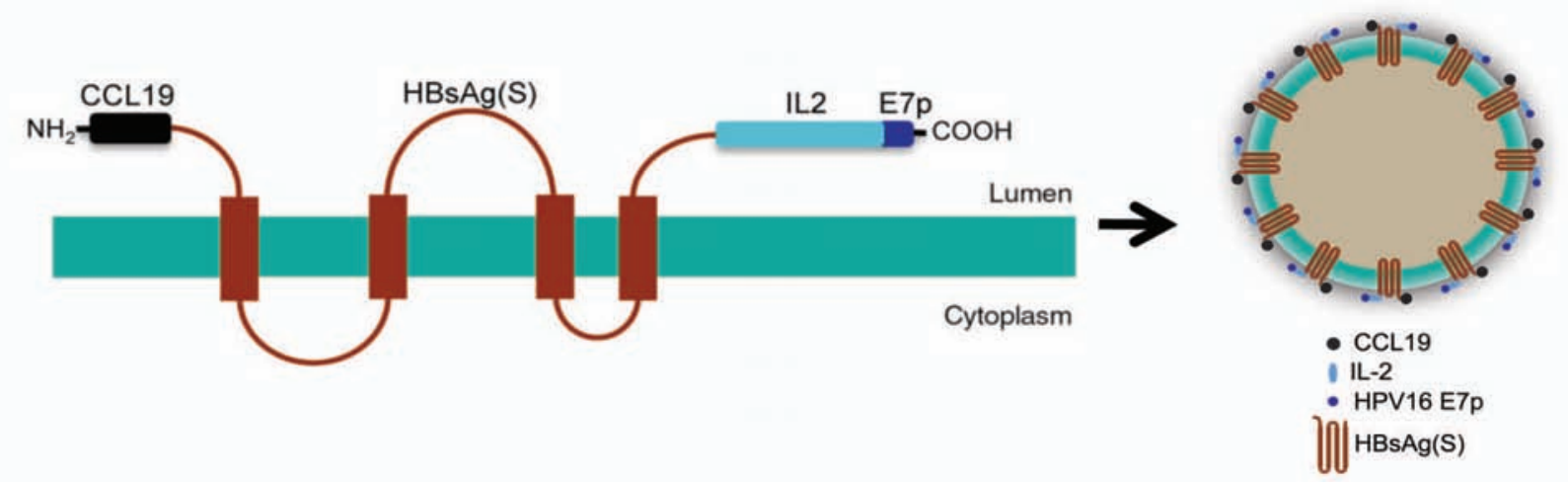

Fig. (1). Schematic representation of the CHI-E7p protein and VLP. The HBsAg(S) protein is represented as it is hypothesized to appear in the membranes of the Golgi apparatus before assembling into VLPs, i.e. a polypeptide with four transmembrane domains with the CCL19 at its N-terminus and IL-2 plus E7p at its C-terminus. On the right, a cartoon of a hypothetical VLP made of CHI-E7p.

yeast $P$. pastoris, subsequently purified over Ni-NTAagarose columns and pooled elution fractions were concentrated using Amicon filters. The resulting purified protein was subjected to negative staining and transmission electron microscopy analysis, which showed VLPs of 20-25 $\mathrm{nm}$ in diameter (Fig. 2b). These purified fractions were used for subsequent functional analyses described below.
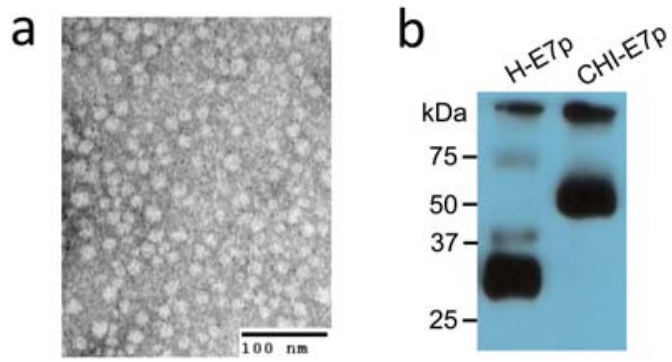

Fig. (2). Analysis of CHI-E7p protein expressed in yeast and human cells. (a) Transmission electron microscopy of negatively stained CHI-E7p VLPs purified by ion metal affinity chromatography (Ni-NTA-agarose columns) from the yeast $P$. pastoris transformed with the pPICZ(A)-CHI-E7p plasmid. The VLPs appear as nanoparticles of 20-25 nm in diameter. (b) Western blot of HEK293 cells transfected transiently with pcDNA3 plasmids encoding either the CHI-E7p or the H-E7p fusion proteins. The cells were lysed and electrophoresed 48 hours after transfection. The proteins were detected with anti-6x His antibodies. The CHI-E7p protein runs with a molecular weight of $\sim 55 \mathrm{kDa}$ while the H-E7p protein appears as a $\sim 28 \mathrm{kDa}$ band.

\section{Characterization of the CHI-E7p Chimeric Protein}

As a first step to test the immunogenic properties of CHIE7p, we examined whether the CCL19 and the IL-2 domains were functional in the context of their linkage with HBsAg(S). First, we tested the ability of the CHI-E7p to attract DCs, as it could be expected if its CCL19 moiety is accessible and can bind to its receptor (CCR7) in mature DCs. We used recombinant CHI-E7p protein produced in the yeast $P$. pastoris and purified by affinity chromatography to perform a transmigration assay using DCs isolated from the bone marrow of HLA-A2 (AAD) transgenic mice and induced to mature with TNF- $\alpha$. As shown in Fig. (3a) the CHI-E7p protein added to the lower chamber of the transwell was able to attract CFSE-labeled DCs in a dosedependent manner, as revealed by the increasing levels of fluorescence measured in the lower chamber. Further, the functionality of the IL-2 present in CHI-E7p was measured using a cell proliferation assay with the CTLL-2 cell line, which depends upon IL-2 for growth. The results of these assays are summarized in Fig. (3b), which shows that addition to the medium of CHI-E7p but not H-E7p stimulated growth of CTLL-2 cells above basal levels, and this effect was dose-dependent. These two sets of results indicate that the two immune-stimulatory domains in the CHI-E7p protein are accessible on the surface of the VLPs and can interact with their respective receptors.

\section{Cytotoxic $T$ Cell Responses in AAD (HLA-A2) Transgenic Mice Immunized with Plasmid Encoding CHI-E7p}

To circumvent the technical complexity of producing large amounts of protein with the appropriate purity grade for immunization studies, we decided to perform our immunization studies using an in vivo DNA transduction protocol to test pcDNA3 plasmids harboring the genes encoding either CHI-E7p or H-E7p. Strong expression of both proteins was observed in transient transfection tests with these plasmids (Fig. 2b) and hence expression was expected to take place also in vivo. Naked plasmid DNA was administered to the mice intramuscularly in three doses (100 $\mu \mathrm{g} / \mathrm{dose})$ at two-week intervals. One week after the last immunization, splenocytes were isolated and the E7-specific CD8+ $\mathrm{T}$ cell responses measured. The results are summarized in Fig. (4a). Immunization with pcDNA3-CHIE7p evoked significantly higher numbers of peptide-specific CD8+ T cells for both E7(11-21) and E7(82-90) peptides compared to mice immunized with pcDNA3-H-E7p.

Further, we analyzed the cytotoxic $\mathrm{T}$ cell responses induced by immunization with the pcDNA3-CHI-E7p and pcDNA3-H-E7p plasmids in the HLA-A2 transgenic mice. Groups of immunized and non-immunized mice were injected intravenously with mixtures of equal numbers of three populations of splenocytes isolated from naïve mice, loaded with the peptides E7(11-20), E7(82-90), or not loaded as control, and labeled with the vital dye CFSE at high, low or intermediate concentrations, respectively. These three populations were analyzed by flow cytometry and quantified 

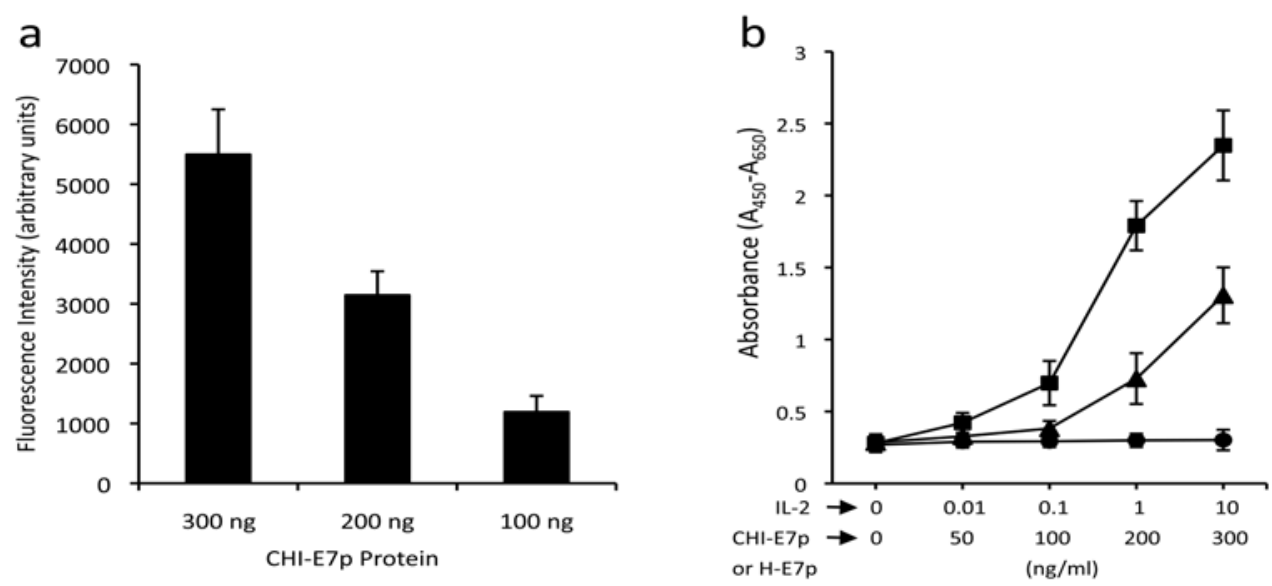

Fig. (3). Functional characterization of the CHI-E7p protein. (a) Transmigration assay. The graph shows the chemotactic activity of the CHIE7p fusion protein on DCs isolated from HLA-A2 (AAD) transgenic mice that were labeled with CFSE and added to the upper chamber of 24-well transwell plates ( $5 \mu \mathrm{m}$ pore size) in medium X-Vivo. The lower chamber contained X-vivo with the indicated concentrations of CHIE7p fusion protein. Fluorescence intensity was measured in a Victor ${ }^{\circledR}$ plate reader. Fluorescence intensity values measured in wells in which no protein was added to the lower chamber were subtracted from those measured in the wells containing CHI-E7p protein. The fluorescence intensity was dose-dependent. (b) Cytokine-dependent proliferation assay. Represented is the proliferation of CTLL-2 cells in response to the CHI-E7p protein. CTLL-2 cells, which depend on IL-2 for growing, were plated in 96-well plates with medium supplemented with IL-2 (squares), CHI-E7p (triangles) or H-E7p (circles) at the indicated concentrations and incubated for 48 hours. Cell proliferation was measured with the WST-1 assay and is given as absorbance at $450 \mathrm{~nm}$ after subtracting the absorbance at a reference wavelength of $650 \mathrm{~nm}$. The CTLL-2 cells only proliferate in the presence of IL-2 or CHI-E7p, but not H-E7p. This experiment was performed twice.
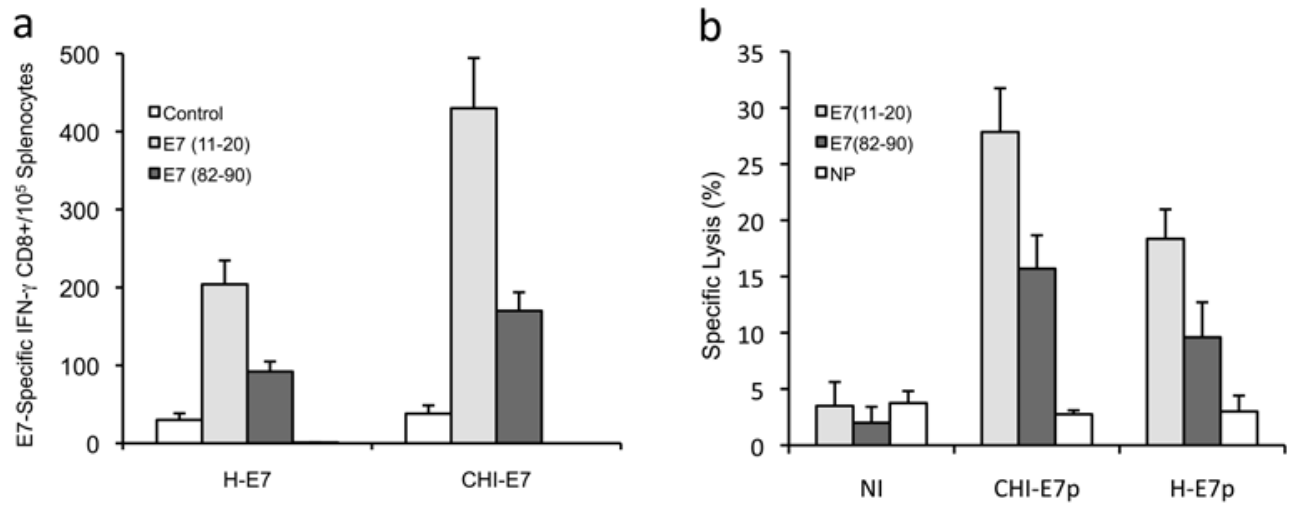

Fig. (4). Detection of HPV16 E7-peptide-specific T cell responses to immunization with plasmid DNA encoding CHI-E7p or H-E7p fusion proteins. (a) Intracellular cytokine staining and flow cytometry analysis of E7 peptide-specific CD8+ T cell responses. Groups of female HLA-A2 (AAD) transgenic mice (5-6/group) were immunized with pcDNA3-CHI-E7p or pcDNA3-HE-7p. Splenocytes were isolated and incubated overnight either with peptide E7(11-20) or E7(82-90) or without peptide (control). After staining with anti-CD8 and anti-IFN- $\gamma$ antibodies, the T cells were analyzed by flow cytometry to detect CD8+/IFN- $\gamma+\mathrm{T}$ cells. The graph shows mean numbers (with s.d.) of IFN$\gamma+$ /E7E7 peptide-specific CD8+ T cells detected per $10^{5} \mathrm{~T}$ cells. (b) Cytotoxic T cell responses induced by immunization against the CHIE7p or H-E7p fusion proteins as determined by antigen-specific kill of indicator cell populations. Female HLA-A2 (AAD) transgenic mice were immunized at 2-week intervals with pcDNA3-CHI-E7p or pcDNA3-HE-7p. Six days after the fourth boost immunization, splenocytes isolated from naïve mice were loaded with peptide E7(11-21) or E7(82-90) or left without peptide (NP), and were subsequently labeled with CFSE at high, low and intermediate concentrations, respectively. Equal numbers of labeled cells were mixed, and $15 \times 10^{6}$ cells of the mixture were injected intravenously into control (non-immunized, NI) and immunized mice. Lymphocytes from spleens and regional lymph nodes were taken $20 \mathrm{~h}$ after injection and analyzed by flow cytometry. The data shown are representative of two experiments performed.

16 hours after being injected. As shown in Fig. (4b) the E7(11-20)/CFSE ${ }^{\text {high }}$ and E7(82-90)/CFSE ${ }^{\text {low }}$ cell populations underwent significant reduction in the immunized mice either with pcDNA3-CHI-E7p or pcDNA3-H-E7p. Nevertheless, significantly higher percentages of specific lysis were observed in the mice immunized with pcDNA3-CHIE7p for both E7(11-20) and E7(82-90) peptides. These data indicated the superior ability of the CHI-E7p construct to induce cytotoxic $\mathrm{T}$ cell responses in the immunized animals.

\section{Tumor Challenge}

We tested whether vaccination with the plasmid pcDNA3-CHI-E7 was able to protect against tumor formation after challenge with TC-1/A2 cells in the HLA-A2 
transgenic mice. TC-1/A2 is an established tumor cell line that expresses constitutively the E7 protein of HPV16 and can process and present E7 antigen via HLA-A2 [11]. The expression of HLA-A2 in the TC-1/A2 cells was tested with an anti-HLA-A2 antibody (not shown). Control nonimmunized mice and those receiving pcDNA3 empty vector developed palpable tumors within 1-2 weeks (Fig. 5). In contrast, $80 \%$ and $40 \%$ of mice vaccinated with pcDNA3-HE7p or pcDNA3-CHI-E7p, respectively, remained tumorfree and in those where a tumor was detected progression was slower compared to the control group (not shown). These results demonstrate the capability of the pcDNA3CHI-E7 construct to elicit HLA-A2-restricted anti-tumor responses in a prophylactic setting and also show that the CHI-E7p fusion protein provides a higher level of protection compared to the H-E7 protein.

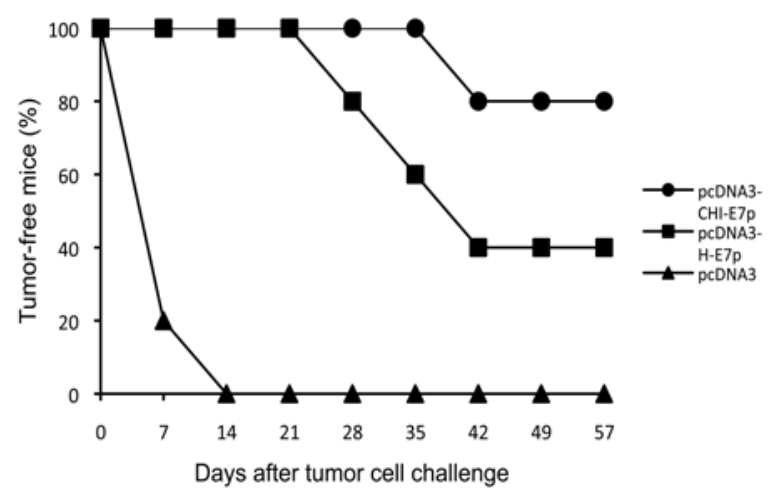

Fig. (5). Tumor protection observed in immunized mice. The graph shows the percentage of tumor-free animals after being challenged with tumor cells. HLA-A2 (AAD) transgenic mice were immunized with either pcDNA3-CHI-E7p or pcDNA3-H-E7p, or injected with pcDNA empty vector. One week after the last immunization the mice were injected s.c. each with $5 \times 10^{4}$ TC-1/A2 cells. Tumor growth was monitored by palpation every 3-4 days. The data shown are from one representative immunization experiment of two performed.

\section{DISCUSSION}

In this study, we sought to investigate the hypothesis that HBsAg(S) could harbor added immune-stimulatory domains at its $\mathrm{N}$-and $\mathrm{C}$-termini and assemble into nanoparticles (VLPs) with enhanced immunogenic potential against a tumor antigen. To this end, as a proof-of-concept, we designed and characterized a complex chimeric protein based on the hepatitis virus $\mathrm{HBsAg}(\mathrm{S})$ envelope protein to which we linked CCL19 at the N-terminus and IL-2 and a HPV16 E7 polytope at its C-terminus (CHI-E7p). When expressed in yeast this construct assembled into nanoparticles (VLPs) of 20-25 $\mathrm{nm}$ as shown by negative staining and transmission electron microscopy (Fig. 2a). This is similar to the size of the wild-type $\operatorname{HBsAg}(\mathrm{S})$ and the $\operatorname{HBsAg}(\mathrm{S})$-E7 VLPs described previously [9].

A strong expression of the CHI-E7p fusion protein was observed in transient transfection tests with HEK293 cells. Western blot analysis of the transfected cells showed a protein of about $55 \mathrm{kDa}$ (Fig. 2b) that was detected with specific antibodies against each of the components of the CHI-E7p fusion protein (except for E7p, because antibodies were not available). This confirmed that the fusion protein was translated as expected and that no proteolytic cleavage occurred.

We next investigated whether the CCL19 and IL-2 domains in the CHI-E7p fusion protein were functional. In our study we used the human CCL19 and IL-2 sequences to construct the CHI-E7p fusion protein. The human sequences were used deliberately in order to test a construct that eventually could be used in humans. Nevertheless, it has been demonstrated that the human CCL19 attracts mouse splenocytes [21] and that human IL-2 promotes proliferation of mouse $\mathrm{T}$ cells with comparable efficiency to mouse IL-2 [22]. Indeed, our results showed that both the CCL19 and the IL-2 moieties in the CHI-E7p fusion protein were functional (Fig. 3).

The strong expression of CHI-E7p protein in our transfection test, which was somehow expected because we used codon-optimized DNA sequences as described previously [14]. encouraged us to pursue immunization studies with naked plasmid DNA injection in HLA-A2 (AAD) transgenic mice. The immunized mice showed E7specific CD8+ T cell responses to both E711-20 and E78290 peptides, which were significantly stronger in mice receiving plasmid expressing the CHI-E7p fusion protein compared to those immunized with the plasmid encoding $\mathrm{H}-$ E7p, a tendency that was confirmed in a cytotoxic assay in vivo (Fig. 4). The CD8+ responses to peptide E7(82-90) were significantly lower than those to E7(11-20), yet above the background levels detected with irrelevant peptides. In contrast, Peng et al. [11] have reported that HLA-A2 mice immunized with a calreticulin-E7 fusion protein did not show a detectable E7(82-90)-specific T cell response. Since we used the same strain of HLA-A2 (AAD) transgenic mice and plasmid immunization too, although with a different protocol, such discrepancy may only be attributed to the kind of construct that we used (VLPs carrying immunestimulatory domains and a polytope instead wild type or mutant E7). Altogether our data suggest that the protection against tumor challenge with TC-1/A2 cells observed in HLA-A2 (AAD) transgenic mice (Fig. 5) immunized with the plasmids of this study relies primarily on the E7(11-20)specific $\mathrm{T}$ cell responses.

In summary, our study has shown that immunization with plasmid encoding VLPs that harbor an HPV16 E7 small polytope and the CCL19 and IL-2 immune-stimulatory domains elicits HLA-A2-restricted E7-specific CD8+ T cell responses, which can prevent tumor growth after challenge with the isogenic, E7-expressing cell line TC-1/A2. To our knowledge this is the first combination of a VLP-forming $\mathrm{HBsAg}(\mathrm{S})$ subunit, tumor antigens and cytokines in the same molecule. These results suggest that such HBsAg-based VLPs may be beneficial for the treatment of HPV-associated neoplasias. However, we are aware of the potential problems posed by restrictions in the host immune responses associated to HPV infection, which might restrict the applicability of such kind of approach in humans. These include local immunosuppression promoted by HR-HPVs by several mechanisms [5], for instance, tolerance to E6 and E7 as a result of their persistent low-level expression, repression of antigen processing and presentation induced by E7 and/or variations in the ability of individuals to generate potent 
antiviral T-cell responses to particular viral epitopes depending on genetic background and HLA type.

Although we have used a prototypic VLP carrying CCL19 and IL-2, other immune-modulators may be used to entrap DCs, stimulate lymphocytes and/or activate natural killer cells. Also, the combinatorial use of VLPs carrying different tumor antigens and diverse immune-stimulatory molecules should be explored. VLPs carrying smaller immune-stimulatory domains might be easier to produce and purify in large amounts. It remains to be investigated whether immunization with purified VLPs instead of plasmid DNA encoding them might have higher immunogenic potential and also whether local rather than systemic immunization might help attract immune-effector cells and thus generate more effective E7-specific T cell responses. Further studies are also required to investigate whether similar strong responses can be obtained against non-viral, endogenous tumor-associated antigens.

$$
\begin{aligned}
& \text { ABBREVIATIONS } \\
& \text { CIN }=\text { Cervical intraepithelial neoplasia } \\
& \text { CCL19 }=\text { Chemokine } \mathrm{CC} \text { ligand } 19 \\
& \mathrm{DC}=\text { Dendritic cell } \\
& \text { CTL = Cytotoxic } \mathrm{T} \text { lymphocyte } \\
& \operatorname{HBs} \operatorname{Ag}(S)=\text { Hepatitis B small surface antigen } \\
& \text { HR-HPV = High-risk human Papillomavirus } \\
& \text { IL-2 = Interleukine-2 } \\
& \text { VLP }=\text { Virus-like particle }
\end{aligned}
$$

\section{CONFLICT OF INTEREST}

The authors confirm that this article content has no conflict of interest.

\section{ACKNOWLEDGEMENTS}

We wish to thank Prof $\mathrm{H}$. zur Hausen for continuous advice and helpful discussions. We are thankful to W. Hunziker for sequencing, W. Weinig for synthesis and purification of oligonucleotides, and K. Müller, D. Pfeiffer, I. Kupin and D. Muth for excellent technical assistance. We are gratefully acknowledged to the staff of the laboratory of animal resources at DKFZ for their constant support.

\section{REFERENCES}

[1] Cid-Arregui A. Prophylactic HPV Vaccines. Open Vaccine J 2009; 2: $123-33$.

[2] Lowy DR, Howley PM. Papillomaviruses. In: Howley PM, Ed. Virology. Philadelphia, USA: Lippincott Williams and Wilkins 2001; pp. 2231-64.

[3] Munger K, Howley PM. Human papillomavirus immortalization and transformation functions. Virus Res 2002; 89(2): 213-28.
[4] zur Hausen H. Papillomaviruses in human cancers. Proc Assoc Am Physicians 1999; 111(6): 581-7.

[5] Cid-Arregui A. Therapeutic vaccines against human papillomavirus and cervical cancer. Open Virol J 2009; 3: 67-83.

[6] Kanodia S, Da Silva DM, Kast WM. Recent advances in strategies for immunotherapy of human papillomavirus-induced lesions. Int J Cancer 2008; 122(2): 247-59.

[7] Su JH, Wu A, Scotney E, et al. Immunotherapy for cervical cancer: Research status and clinical potential. Bio Drugs 2010; 24(2): 10929.

[8] Kruger DH, Ulrich R, Gerlich WH. Chimeric virus-like particles as vaccines. Biol Chem 1999; 380(3): 275-6.

[9] Baez-Astua A, Herraez-Hernandez E, Garbi N, et al. Low-dose adenovirus vaccine encoding chimeric hepatitis B virus surface antigen-human papillomavirus type $16 \mathrm{E} 7$ proteins induces enhanced E7-specific antibody and cytotoxic T-cell responses. J Virol 2005; 79(20): 12807-17.

[10] Lin KY, Guarnieri FG, Staveley-O'Carroll KF, et al. Treatment of established tumors with a novel vaccine that enhances major histocompatibility class II presentation of tumor antigen. Cancer Res 1996; 56(1): 21-6.

[11] Peng S, Trimble C, He L, et al. Characterization of HLA-A2restricted HPV-16 E7-specific CD8(+) T-cell immune responses induced by DNA vaccines in HLA-A2 transgenic mice. Gene Ther 2006;13(1): 67-77.

[12] Gillis S, Smith KA. Long term culture of tumour-specific cytotoxic T cells. Nature 1977; 268(5616):154-6.

[13] Newberg MH, Smith DH, Haertel SB, Vining DR, Lacy E, Engelhard VH. Importance of MHC class 1 alpha2 and alpha3 domains in the recognition of self and non-self MHC molecules. J Immunol 1996; 156(7): 2473-80.

[14] Cid-Arregui A, Juarez V, zur Hausen H. A synthetic E7 gene of human papillomavirus type 16 that yields enhanced expression of the protein in mammalian cells and is useful for DNA immunization studies. J Virol 2003; 77(8): 4928-37.

[15] Ressing ME, Sette A, Brandt RM, et al. Human CTL epitopes encoded by human papillomavirus type 16 E6 and E7 identified through in vivo and in vitro immunogenicity studies of HLAA*0201-binding peptides. J Immunol 1995; 154(11): 5934-43.

[16] Inaba K, Inaba M, Romani N, et al. Generation of large numbers of dendritic cells from mouse bone marrow cultures supplemented with granulocyte/macrophage colony-stimulating factor. J Exp Med 1992; 176(6): 1693-702.

[17] Cyster JG. Chemokines and the homing of dendritic cells to the T cell areas of lymphoid organs. J Exp Med 1999; 189(3): 447-50.

[18] Sallusto F, Mackay CR, Lanzavecchia A. The role of chemokine receptors in primary, effector, and memory immune responses. Annu Rev Immunol 2000; 18: 593-620.

[19] Yanagihara S, Komura E, Nagafune J, Watarai H, Yamaguchi Y. EBI1/CCR7 is a new member of dendritic cell chemokine receptor that is up-regulated upon maturation. J Immunol 1998; 161(6): 3096-102.

[20] Rosenberg SA. Interleukin-2 and the development of immunotherapy for the treatment of patients with cancer. Cancer J Sci Am 2000; 6 (Suppl 1): S2-7.

[21] Kim CH, Pelus LM, White JR, Applebaum E, Johanson K, Broxmeyer HE. CK $\beta-11 /$ Macrophage Inflammatory Protein$3 \beta /$ EBI1-Ligand Chemokine Is an Efficacious Chemoattractant for T and B Cells. J Immunol 1998; 160(5): 2418-24.

[22] Mosmann TR, Yokota T, Kastelein R, Zurawski SM, Arai N, Takebe Y. Species-specificity of T cell stimulating activities of IL 2 and BSF-1 (IL 4): comparison of normal and recombinant, mouse and human IL 2 and BSF-1 (IL 4). J Immunol1987; 138(6): 1813-6. 